\title{
Determinants of outcome in operatively and non-operatively treated Weber-B ankle fractures
}

\author{
E. M. Van Schie-Van der Weert • \\ E. M. M. Van Lieshout • M. R. De Vries • \\ M. Van der Elst $\cdot$ T. Schepers
}

Received: 16 April 2011 / Published online: 30 September 2011

(C) The Author(s) 2011. This article is published with open access at Springerlink.com

\begin{abstract}
Introduction Treatment of ankle fractures is often based on fracture type and surgeon's individual judgment. Literature concerning the treatment options and outcome are dated and frequently contradicting. The aim of this study was to determine the clinical and functional outcome after AO-Weber B-type ankle fractures in operatively and conservatively treated patients and to determine which factors influenced outcome.

Patients and methods A retrospective cohort study in patients with a AO-Weber B-type ankle fracture. Patient, fracture and treatment characteristics were recorded. Clinical and functional outcome was measured using the Olerud-Molander Ankle Score (OMAS), the American Orthopaedic Foot and Ankle Society ankle-hindfoot score (AOFAS) and a Visual Analog Score (VAS) for overall satisfaction (range 0-10).

Results Eighty-two patients were treated conservatively and 103 underwent operative treatment. The majority was female. Most conservatively treated fractures were AO-Weber B1.1 type fractures. Fractures with fibular displacement (mainly AO type B1.2 and Lauge-Hansen type SER-4) were predominantly treated operatively. The
\end{abstract}

E. M. Van Schie-Van der Weert

Department of Emergency Medicine,

Reinier de Graaf Groep Delft, Delft, The Netherlands

E. M. M. Van Lieshout - T. Schepers ( $\square)$

Department of Surgery-Traumatology, Erasmus MC, University Medical Center Rotterdam, Room H-822k, P.O. Box 2040, 3000 CA Rotterdam, The Netherlands e-mail: t.schepers@erasmusmc.nl

M. R. De Vries · M. Van der Elst - T. Schepers Department of Surgery and Traumatology,

Reinier de Graaf Groep Delft, Delft, The Netherlands outcome scores in the non-operative group were OMAS 93, AOFAS 98, and VAS 8. Outcome in this group was independently negatively affected by age, affected side, BMI, fibular displacement, and duration of plaster immobilization. In the surgically treated group, the OMAS, AOFAS, and VAS scores were 90, 97, and 8, respectively, with outcome negatively influenced by duration of plaster immobilization.

Conclusion Treatment selection based upon stability and surgeon's judgment led to overall good clinical outcome in both treatment groups. Reducing the cast immobilization period may further improve outcome.

Keywords Ankle Fracture Outcome - Operative . Non-operative

\section{Introduction}

Ankle fractures are one of the most common fractures encountered in the Emergency Department. They represent approximately $10 \%$ of all fractures [7] and previous studies suggest that their incidence is rising [8]. Currently operative treatment dominates the literature on ankle fractures; however, conservative treatment of stable ankle fractures is gaining more interest. Studies comparing operative and conservative treatment are dated and contradicted in their conclusions. For example, Makwana et al. [17] found superior outcome in patients over 55 years of age managed by open reduction and internal fixation (ORIF) compared with closed treatment. Beauchamp et al. [3], on the other hand, reported little difference in functional outcome after conservative or operative treatment of displaced ankle fractures in patients over 50 years of age. Table 1 summarizes the results of previous studies comparing the 
Table 1 Literature overview of studies comparing operative versus conservative treatment of ankle fractures
$P$ Prospective, $R$ retrospective, $R C T$ randomized controlled trial, SER\# supination exorotation fracture, $O$ ORIF, $\mathrm{C}$ conservative treatment,

N.A. not available

\begin{tabular}{|c|c|c|c|c|c|c|c|}
\hline & $\begin{array}{l}\text { Study } \\
\text { design }\end{array}$ & $\begin{array}{l}\text { No. of } \\
\text { patients }\end{array}$ & $\begin{array}{l}\text { ORIF } \\
(N)\end{array}$ & $\begin{array}{l}\text { Cons } \\
(N)\end{array}$ & $\begin{array}{l}\text { Average } \\
\text { age } \\
\text { (years) }\end{array}$ & $\begin{array}{l}\text { Mean follow-up } \\
\text { (months) }\end{array}$ & $\begin{array}{l}\text { Clinical Outcome } \\
\text { favors ORIF? - no } \\
+ \text { yes; = equal }\end{array}$ \\
\hline Dietrich et al. [9] & $\mathrm{P}$ & 57 & 23 & 34 & 49 & 17 & - \\
\hline Makwana et al. [17] & RCT & 43 & 22 & 21 & $>55$ & 27 & + \\
\hline Anand 1993 [1] & $\mathrm{R}$ & 80 & 39 & 41 & $>60$ & $28(\mathrm{O}), 25.5(\mathrm{C})$ & + \\
\hline Rowley et al. [22] & RCT & 42 & 20 & 22 & $16-70$ & 5 & $=$ \\
\hline Bauer et al. [2] & $\mathrm{RCT}$ & 92 & 43 & 49 & 50 & 84 & $=/$ early + \\
\hline Philips et al. [21] & RCT & 71 & 45 & 26 & 41 & 42 & + \\
\hline $\begin{array}{l}\text { Beauchamp et al. } \\
\text { [3] }\end{array}$ & $\mathrm{R}$ & 126 & 71 & 55 & $>50$ & 24 & $=$ \\
\hline Tunturi et al. [26] & $\mathrm{R}$ & 239 & 124 & 115 & 46 & 36 & $\begin{array}{c}=(\text { Depending on } \\
\text { radiological } \\
\text { result })\end{array}$ \\
\hline Yde 1980 [29] & $\mathrm{R}$ & 69 & 34 & 35 & $15-49$ & $36-120$ & $=($ in SER2) \\
\hline Yde 1980 [30] & $\mathrm{R}$ & 89 & 60 & 29 & $15-75$ & $36-120$ & + (in SER4) \\
\hline Eventov 1978 [12] & $\mathrm{R}$ & 200 & 101 & 99 & $16-87$ & 48 & - \\
\hline Malka 1969 [18] & $\mathrm{R}$ & 50 & 25 & 25 & N.A. & 19.4 & $=1+$ \\
\hline Wilson 1966 [27] & $\mathrm{R}$ & 55 & 28 & 27 & 45.3 & 97 & $=$ \\
\hline
\end{tabular}

results of conservative and operative treatment of displaced ankle fractures.

The decision whether to operate or not is often based upon the Arbeitsgemeinschaft Osteosynthesefragen (AO) modifications of Weber's classification and the LaugeHansen classification [26], but is also highly dependent upon the surgeon's individual judgment on displacement. There is consensus that undisplaced Weber A-type fractures rarely require operative treatment, and that Weber C-type or grossly displaced fractures are unstable by nature and therefore require surgery. The gray area of treatment lies mainly with the minimally displaced Weber B-type ankle fractures.

The primary aim of this study was to determine the outcome of operatively and conservatively treated patients with an AO-Weber B-type ankle fracture. The secondary aim was to gain insight in various factors influencing outcome.

\section{Materials and methods}

\section{Patients}

All consecutive patients aged between 16 and 65 years, treated between January 2004 and July 2009, with a AO-Weber B-type ankle fracture were included in this retrospective study. Minimum follow-up was 6 months. Patient characteristics (i.e., age, gender, diabetes, and smoking habits), fracture characteristics (i.e., fracture side, displacement, AO-Weber fracture type [14], Lauge-Hansen classification [16] and number of malleoli involved), and treatment characteristics (i.e., operative vs. conservative) were recorded from the patient files, operation reports, and the picture archiving and communication system (PACS: Kodak Carestream ${ }^{(}$). This study was performed with the approval of the local medical ethics committee.

Treatment protocol

Treatment was based upon patient characteristics (co-morbidities, level of activity), fracture characteristics (number of fractured malleoli, displacement, and stability) and on surgeons preference concerning the threshold of displacement of the distal fibular fragment and shortening of the fibula, which was usually at $2 \mathrm{~mm}$. Most fractures were initially treated in a non-weight bearing plaster backslap and evaluated at the outpatient department within 1 week.

Conservative treatment concerned immobilization in a below-knee plaster cast usually for 6 weeks. During the application of the cast, a manual reduction was tried by reversing the trauma-mechanism using the Lauge-Hansen classification. Immediately after application of the cast at the Emergency Department, radiographs were taken to confirm that reduction was adequate. During the final 3 weeks, the patients were allowed partial or full weight bearing in the cast.

ORIF was performed according to the AO-guidelines under fluoroscopic control, and performed by residents under supervision of a staff member [16]. All patients received preoperative antibiotic prophylaxis (i.e., third generation cephalosporin). After surgery, the ankle was usually immobilized with a below-knee plaster cast for 
6 weeks. Weight bearing in the cast was usually allowed after 2 weeks [24].

In both treatment groups, radiographs were taken at 2 and 6 weeks to ensure that there was no loss of reduction and to monitor fracture healing. During immobilization in plaster cast, all patients were treated with low-molecularweight heparin, once daily, as thrombosis prophylaxis. After cast removal, patients commenced weight bearing under supervision of a physiotherapist.

\section{Radiographic assessment}

Radiographic data were obtained from the radiographs taken immediately after the injury and immediately after operative repair. A total of four of the pre-operative radiographs were missing rendering it impossible to classify these fractures. Dislocation was measured as lateral displacement of the fibula at the level of the tibial plafond and the distal anteromedial border of the fibula (Mueller nose), and at the Medial Clear Space (MCS) at $10 \mathrm{~mm}$ below the tibial plafond. Fracture classification according to AO-Weber and Lauge-Hansen was performed by two observers (ES, TS). In case of differences consensus was met after discussion. MCS and dislocation at the Mueller nose as measure of lateral displacement of the fibula were calculated on the same radiographs.

\section{Outcome measurement}

Outcome was measured using standard questionnaires, which was sent in January 2010, and consisted of the Olerud-Molander Ankle Score (OMAS), the American Orthopaedic Foot and Ankle Society ankle-hindfoot score (AOFAS), and a single question Visual Analog Scale (VAS) for patient satisfaction with outcome. A reminder was sent after 4 weeks.

The OMAS is a self-administered patient questionnaire with a score of zero (totally impaired) to 100 (completely unimpaired) and is based on nine different items: pain, stiffness, swelling, stair climbing, running, jumping, squatting, supports and work/activities of daily living [20].

The AOFAS ankle hindfoot score was introduced in 1994 by Kitaoka et al. [15]. and includes nine questions on pain, activity and functional limitations, walking distance, difficulties with different terrains, gait abnormality, sagittal range of motion at the ankle and range of motion at the subtalar joint, stability, and alignment (whether or not plantigrade). The nine questions relate to three components: pain (one question; 40 points), function (seven questions; 50 points), and alignment (one question; 10 points) leading to a total possible score of 100 points. The question related to alignment and range of motion was completed by a physician based upon patient files and radiographs; the other questions were completed by the patient.

A Visual Analog Scale was used to measure overall satisfaction of patients with outcome (range 0-10).

Statistical analysis

The statistical analysis was performed using the Statistical Package for the Social Sciences (SPSS) version 16.0 (SPSS, Chicago, IL, USA). The Kolmogorov-Smirnov test was used to test the normality of the numeric data. The Levene's test was applied to assess homogeneity of variance between data. Since most numeric variables did not show normal distribution or equal variance, all items were regarded as nonparametric for the statistical analysis. Mann-Whitney $U$ tests were performed to assess statistical differences in OMAS, AOFAS and VAS scores between subgroups. Numeric data are expressed as medians with $\mathrm{P}_{25}-\mathrm{P}_{75}$; categoric data are shown as numbers with percentages. Logistic regression models were developed to correct for gender, age, diabetes, smoking habits, BMI, fracture-type, and fracture-dislocation. A $p$ value $<0.05$ was taken as level of statistical significance.

\section{Results}

\section{Baseline characteristics}

A total of 185 (response rate $71 \%$ ) out of 261 approached patients returned the questionnaire; 82 patients were treated conservatively (response rate 69\%) by immobilization in a plaster cast and 103 patients had undergone surgical treatment (response rate $72 \%$ ). Table 2 shows the baseline characteristics of the two groups. Most patients were female $(61.1 \%)$, and the median age at trauma was 50.6 years. The median follow up time was 46 months. Baseline characteristics were similar in both groups, with duration of plaster cast immobilization being the single exception. Almost all patients were immobilized in a plaster cast (96.8\%), $94.2 \%$ in the operatively treated group and $100 \%$ in the conservatively treated group $(p=0.035)$.

In the conservatively treated group the median age was 50.1 years, median FU 49 months. Diabetes was present in $6.1 \%$ of the patients and $26.8 \%$ had a smoking habit. In $40.2 \%$, the right ankle was fractured. Immobilization in a plaster cast was for a median of 6 weeks $\left(\mathrm{P}_{25}-\mathrm{P}_{75}\right.$, 6-8 weeks).

In the operatively treated group the median age was 50.7 years, median FU 43 months. Less people (19.4\%) were smokers compared to the conservatively treated group. The right side was involved in $51 \%$ of the fractures. The syndesmosis was injured in 16 cases $(15.5 \%)$. 
Table 2 Baseline

characteristics

$P$ values $<0.05$ are considered statistically significant

Data are shown as ${ }^{\text {a }}$ numbers with the percentage between brackets or as ${ }^{\mathrm{b}}$ median with the $\mathrm{P}_{25}-\mathrm{P}_{75}$ given between brackets

Data are analyzed using a ${ }^{\mathrm{c}} \mathrm{Chi}$ Square analysis or ${ }^{\mathrm{d}}$ MannWhitney $U$ test

\begin{tabular}{lcccc}
\hline & Total & Conservative & Operative & $p$ value \\
\hline$N$ & 185 & 82 & 103 & \\
Males $^{\mathrm{a}}$ & $72(38.9)$ & $33(40.2)$ & $39(37.9)$ & $0.763^{\mathrm{c}}$ \\
Age $\left(\right.$ years $^{\mathrm{b}}$ & $50.6(37.9-58.7)$ & $50.1(35.7-58.2)$ & $50.7(41.1-60.5)$ & $0.258^{\mathrm{d}}$ \\
BMI $\left(\mathrm{kg} / \mathrm{m}^{2}\right)^{\mathrm{b}}$ & $25.5(23.2-28.7)$ & $24.8(22.8-28.3)$ & $26(23.9-29.4)$ & $0.098^{\mathrm{d}}$ \\
Right side affected $^{\mathrm{a}}$ & $97(54.4)$ & $44(53.7)$ & $53(51.5)$ & $0.770^{\mathrm{c}}$ \\
Cigarette smoker $^{\mathrm{a}}$ & $42(22.7)$ & $22(26.8)$ & $20(19.4)$ & $0.289^{\mathrm{c}}$ \\
Diabetes $^{\mathrm{a}}$ & $11(5.9)$ & $5(6.1)$ & $6(5.8)$ & $1.000^{\mathrm{c}}$ \\
Follow-up (months) $^{\mathrm{b}}$ & $46(31-62)$ & $49(33-64)$ & $43(29-60)$ & $0.155^{\mathrm{d}}$ \\
Cast immobilization $^{\mathrm{a}}$ & $179(96.8)$ & $82(100)$ & $97(94-102)$ & $0.035^{\mathrm{c}}$ \\
Immobilization (weeks) $^{\mathrm{b}}$ & $6(6-8)$ & $6(6-8)$ & $6(6-8)$ & $0.412^{\mathrm{d}}$ \\
\hline
\end{tabular}

A

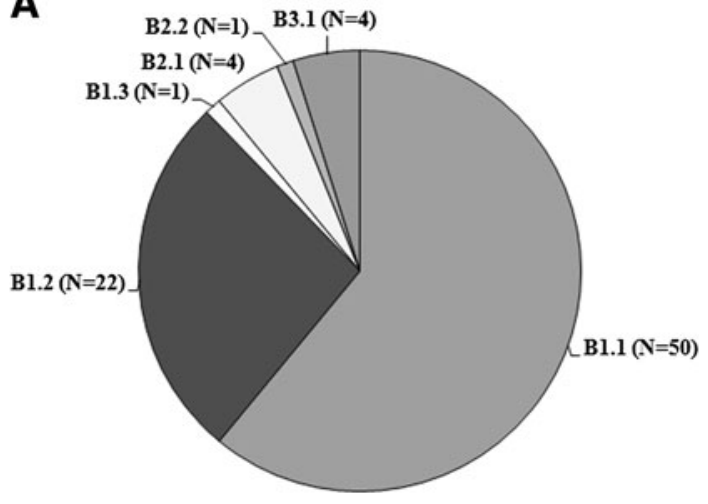

B

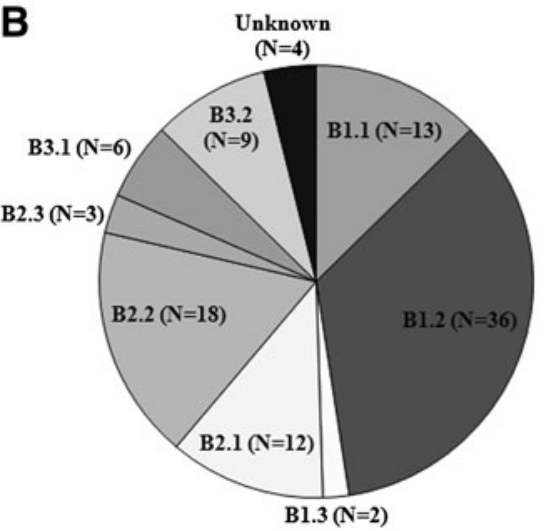

Fig. 1 Fracture type distribution as classified according to AO-Weber; a conservatively treated patients, $\mathbf{b}$ operatively treated patients

\section{Radiographic data}

Radiographic results were determined per treatment modality (Figs. 1, 2; Table 3). In the conservatively treated group, most fractures involved were type AO-B1.1 (61\%) and LH-SER2 $(81.7 \%)$. In $92.7 \%$, there was only unimalleolar involvement. The median fibular dislocation was $0 \mathrm{~mm}$. In the operatively treated group most injuries were type AO-B1.2 (35\%) and LH-SER4 (50.5\%) fractures. Fibular displacement was frequently a reason for ORIF (median $1.39 \mathrm{~mm}$ ).

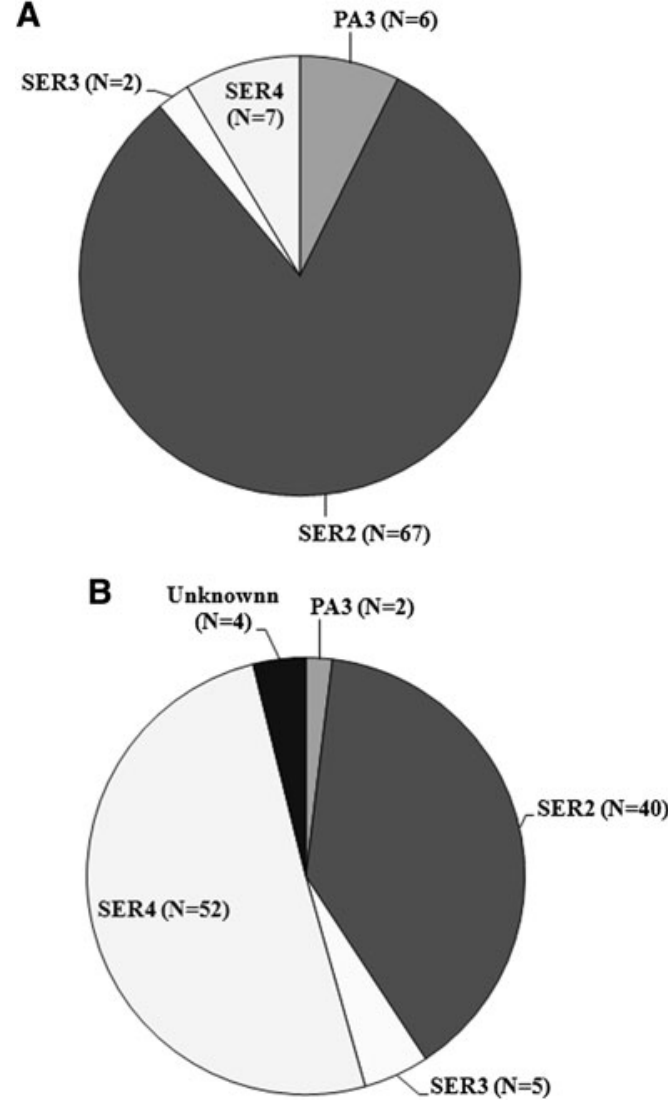

Fig. 2 Fracture type distribution as classified according to LaugeHansen; a conservatively treated patients, b operatively treated patients

\section{Outcome measurement}

In the conservatively treated patients the median OMAS score was 93 points, AOFAS score was 98 points, and the VAS score was 8 points. The operated patient group reported a median OMAS, AOFAS, and VAS score of 90 , 97 , and 8 points, respectively.

Spearman rank correlation showed a statistically significant inverse correlation between BMI and outcome for 
Table 3 Radiographic findings

\begin{tabular}{|c|c|c|c|c|}
\hline Variable & Total & Conservative & Operative & $p$ value \\
\hline \multicolumn{5}{|l|}{ Malleoli involved $^{\mathrm{a}}$} \\
\hline Unimalleolar & $145(78.4)$ & 76 (92.7) & $69(67.0)$ & \multirow[t]{3}{*}{$<0.001^{\mathrm{C}}$} \\
\hline Bimalleolar & $23(12.4)$ & $4(4.9)$ & $19(18.4)$ & \\
\hline Trimalleolar & $17(9.2)$ & $2(2.4)$ & 15 (14.6) & \\
\hline Luxation $^{\mathrm{a}}$ & $12(6.5)$ & $0(0)$ & $12(11.7)$ & $<0.001^{\mathrm{c}}$ \\
\hline \multicolumn{5}{|l|}{ Pre-operative } \\
\hline $\operatorname{MCS}(\mathrm{mm})^{\mathrm{b}}$ & $2.85(2.26-3.90)$ & $2.53(2.19-3.06)$ & $3.66(2.32-5.93)$ & $<0.001^{\mathrm{d}}$ \\
\hline Fibular displacement $(\mathrm{mm})^{\mathrm{b}}$ & $1.08(0.00-1.83)$ & $0.00(0.00-1.48)$ & $1.39(0.66-2.38)$ & $<0.001^{\mathrm{d}}$ \\
\hline \multicolumn{5}{|l|}{ Post-operative } \\
\hline $\operatorname{MCS}(\mathrm{mm})^{\mathrm{b}}$ & $2.58(2.16-3.12)$ & N.A. & $2.58(2.16-3.12)$ & \multirow[t]{2}{*}{ N.A. } \\
\hline Fibular displacement $(\mathrm{mm})^{\mathrm{b}}$ & $0.00(0.00-0.43)$ & N.A. & $0.00(0.00-0.43)$ & \\
\hline
\end{tabular}

both groups. Higher BMI correlated with a worse functional outcome as shown for the AOFAS (Spearman rank correlation coefficient $\left.R_{\mathrm{s}}=-0.249, p=0.001\right)$, OMAS $\left(R_{\mathrm{s}}=-0.210, p=0.005\right), \quad$ and $\operatorname{VAS} \quad\left(R_{\mathrm{s}}=-0.229\right.$, $p=0.002)$.

Multivariable analysis of the conservatively treated patients (Table 4A) showed that an increase in age had an inverse affect on the OMAS, a fracture of the right side gave a lower AOFAS, an increase in distal fibular displacement measured at the Mueller nose resulted in a lower VAS, a higher BMI gave a lower AOFAS, or a longer period of cast immobilization had a significant negative effect on the OMAS. Multivariate analysis of the operatively treated patients (Table 4B) showed that an increase in plaster immobilization gave a worse result on the OMAS and AOFAS score.

No significant correlation could be detected between the number of malleoli involved, smoking or diabetes and functional outcome in both groups.

\section{Discussion}

The aim of this study was to determine the outcome of operatively and conservatively treated AO-Weber B-type ankle fractures, and to gain insight into the factors affecting outcome. The data revealed that the degree of fibular displacement played a significant role with respect to the decision whether to operate or not. This is reflected in the finding that most AO-B1.1 and SER-2 type ankle fractures (without fibular displacement) were treated conservatively with a below-knee cast for 6 weeks. These results are in agreement with the results of Beauchamp et al. [3] and Yablon et al. [28], who concluded that reduction and stable fixation of the lateral malleolus is the key in internal fixation. Functional outcome in this group was good, with a median OMAS score of 93 points, AOFAS score of 98 points, and VAS score of 8 points. Good outcomes were also seen in the study of Dietrich et al. [9], who found that functional treatment of stable Weber-B fractures appeared to be superior to surgery.

Only four randomized trials comparing operative treatment with closed reduction and casting have been published [2, 17, 21, 22]. No significant differences in functional outcome between the two treatment regimens were reported in any of these studies; however, there was a significant loss of follow up in the studies of Philips et al. [21] and Makwana et al. [17]. Bauer et al. [2] showed no difference at the long term between patients treated surgically or by closed reduction and casting, but the surgical group recovered quicker. Rowley on the other hand, found that surgically treated patients took longer to recover normal movement and gait [22]. Philips showed better radiological outcomes in operatively treated patients, but the clinical outcomes were the same in both groups [21]. Makwana et al. [17] showed functional advantage for operatively treated patients.

The findings above suggest that stable fracture might be treated well conservatively. However, unstable fractures fare better with operative treatment $[13,23]$. In the current study, operatively treated patient group, most ankle fractures were of the Weber type b1.2 and unstable LaugeHansen type SER-4. All ankle fracture-dislocations were operated on. Functional outcome in this operated group was similar to the conservatively treated patients, with a median OMAS, AOFAS, and VAS score of 97, 90, and 8 points, respectively. This data would suggest that if there is minimal displacement of the distal fibula conservative management is acceptable in stable ankle fractures. ORIF would be indicated for unstable, displaced ankle fractures. This is in agreement with previous reports by Michelson and by Burwell et al. who showed that accurate reduction and fixation of ankle fractures led to a rapid return of function [6, 19]. 
Table 4 Multivariable analysis of factors influencing outcome in patients treated A conservatively or B operatively

\begin{tabular}{|c|c|c|c|c|c|c|}
\hline \multirow[t]{2}{*}{ Variable } & \multicolumn{2}{|l|}{ OMAS } & \multicolumn{2}{|l|}{ AOFAS } & \multicolumn{2}{|l|}{ VAS } \\
\hline & Beta $(95 \% \mathrm{CI})$ & $P$ & Beta $(95 \% \mathrm{CI})$ & $P$ & Beta $(95 \% \mathrm{CI})$ & $P$ \\
\hline \multicolumn{7}{|c|}{ A Conservatively treated patients } \\
\hline Follow-up duration & $0.021(-0.184 ; 0.220)$ & 0.859 & $0.112(-0.117 ; 0.333)$ & 0.341 & $0.114(-0.012 ; 0.034)$ & 0.344 \\
\hline Age & $-0.288(-0.621 ;-0.044)$ & 0.025 & $-0.152(-0.518 ; 0.123)$ & 0.224 & $-0.124(-0.049 ; 0.016)$ & 0.327 \\
\hline Gender & $0.072(-6.562 ; 11.257)$ & 0.600 & $-0.060(-12.107 ; 7.713)$ & 0.443 & $0.0522(-0.814 ; 1.193)$ & 0.708 \\
\hline Affected side & $-0.198(-13.876 ; 1.108)$ & 0.094 & $-0.241(-17.109 ;-0.442)$ & 0.039 & $-0.170(-1.460 ; 0.228)$ & 0.150 \\
\hline Number of malleoli & $-0.206(-18.053 ; 0.941)$ & 0.077 & $-0.149(-17.693 ; 3.677)$ & 0.195 & $-0.113(-1.616 ; 0.562)$ & 0.338 \\
\hline Pre-MCS & $0.098(-4.034 ; 8.878)$ & 0.456 & $-0.019(-7.007 ; 6.662)$ & 0.886 & $0.020(-0.672 ; 0.783)$ & 0.879 \\
\hline Pre-Mueller & $-0.209(-8.129 ; 0.662)$ & 0.095 & $-0.212(-9.160 ; 0.618)$ & 0.086 & $-0.276(-1.046 ;-0.055)$ & $\mathbf{0 . 0 3 0}$ \\
\hline BMI & $-0.179(-1.222 ; 0.160)$ & 0.130 & $-0.360(-1.973 ;-0.436)$ & 0.003 & $-0.215(-0.149 ; 0.006)$ & 0.072 \\
\hline Smoking & $-0.217(-16.314 ; 0.567)$ & 0.067 & $-0.170(-16.351 ; 2.425)$ & 0.143 & $-0.086(-1.302 ; 0.600)$ & 0.464 \\
\hline Diabetes & $0.029(-14.646 ; 18.760)$ & 0.807 & $0.076(-12.458 ; 24.700)$ & 0.513 & $-0.126(-2.888 ; 0.875)$ & 0.289 \\
\hline Immobilization duration & $-0.371(-4.476 ;-0.849)$ & 0.004 & $-0.076(-2.636 ; 1.398)$ & 0.542 & $-0.247(-0.403 ; 0.006)$ & 0.057 \\
\hline \multicolumn{7}{|c|}{ B Operatively treated patients } \\
\hline Follow-up duration & $-0.089(-0.324 ; 0.064)$ & 0.513 & $-0.089(-0.341 ; 0.173)$ & 0.517 & $-0.005(-0.022 ; 0.021)$ & 0.972 \\
\hline Age & $-0.073(-0.436 ; 0.252)$ & 0.595 & $-0.058(-0.438 ; 0.287)$ & 0.679 & $-0.096(-0.041 ; 0.020)$ & 0.489 \\
\hline Gender & $0.065(-6.895 ; 11.573)$ & 0.614 & $0.164(-3.601 ; 15.872)$ & 0.212 & $0.056(-0.639 ; 0.990)$ & 0.668 \\
\hline Affected side & $-0.141(-13.503 ; 3.706)$ & 0.259 & $-0.105(-12.881 ; 5.264)$ & 0.404 & $-0.145(-1.202 ; 0.317)$ & 0.249 \\
\hline Luxation & $-0.087(-52.265 ; 26.028)$ & 0.505 & $0.086(-27.743 ; 54.808)$ & 0.515 & $0.184(-1.006 ; 5.903)$ & 0.162 \\
\hline Number of malleoli & $-0.191(-13.527 ; 1.593)$ & 0.120 & $-0.229(-15.361 ; 0.447)$ & 0.064 & $-0.190(-1.189 ; 0.145)$ & 0.123 \\
\hline Pre-MCS & $0.057(-1.384 ; 2.091)$ & 0.685 & $0.091(-1.248 ; 2.415)$ & 0.526 & $0.032(-0.136 ; 0.171)$ & 0.822 \\
\hline Pre-Mueller & $0.141(-1.612 ; 5.188)$ & 0.297 & $-0.002(-3.606 ; 3.564)$ & 0.991 & $-0.02(-0.322 ; 0.278)$ & 0.883 \\
\hline Post-MCS & $0.048(-3.796 ; 5.659)$ & 0.695 & $-0.08(-6.583 ; 3.385)$ & 0.524 & $-0.101(-0.588 ; 0.247)$ & 0.417 \\
\hline Post-Mueller & $-0.158(-15.490 ; 3.534)$ & 0.214 & $0.066(-7.412 ; 12.647)$ & 0.604 & $-0.021(-0.908 ; 0.771)$ & 0.871 \\
\hline BMI & $-0.209(-1.921 ; 0.307)$ & 0.153 & $-0.198(-1.971 ; 0.379)$ & 0.181 & $-0.187(-0.162 ; 0.035)$ & 0.202 \\
\hline Smoking & $-0.039(-12.991 ; 9.554)$ & 0.762 & $0.091(-7.645 ; 16.127)$ & 0.478 & $0.125(-0.505 ; 1.485)$ & 0.329 \\
\hline Diabetes & $0.042(-16.665 ; 23.186)$ & 0.745 & $0.129(-10.613 ; 31.406)$ & 0.326 & $0.133(-0.857 ; 2.660)$ & 0.309 \\
\hline Immobilization duration & $-0.368(-4.063 ;-0.515)$ & 0.012 & $-0.375(-4.307 ;-0.565)$ & 0.012 & $-0.286(-0.313 ; 0.001)$ & 0.051 \\
\hline
\end{tabular}

BMI Body mass index, AOFAS American Orthopaedic Foot Ankle Society ankle-hindfoot score, OMAS Olerud-Molander Ankle Score, VAS Visual Analog Scale, MCS Medial Clear Space, pre-Mueller dislocation of the distal fibular fragment pre-operative; post-Mueller dislocation of the distal fibular fragment post-operative

$P$-values printed in bold show statistical significant associations

Overall, age, affected side, fibular displacement at the 'Mueller nose', BMI, and immobilization duration negatively affected outcome in conservatively treated patients. In operated patients, only the immobilization duration was negatively associated with functional outcome. Similar results were shown by Ebraheim et al. [10], who found worse results in patients with a Weber type-C fracture who were treated with ORIF and obesity or diabetes. The latter could not be confirmed in the present study. Boddenberg [4] concluded that ankle fractures in patients with diabetes heal with a significant delay. In a study of Egol et al. [11], younger age, male gender, and absence of diabetes were predictive of improved functional recovery at 1 year following ankle fracture surgery. Our data do not support such a correlation between gender and diabetes with functional outcome as measured with the OMAS, AOFAS, or VAS. The number of fractured malleoli did not affect outcome in the current study, compared with the results by Broos et al. [5].

The treatment protocol used in the current study is very similar to the protocol used in the other studies [9, 17, 24, 25]. Especially in the operative group, patients were left non-weight bearing for at least 2 weeks, depending on wound-healing. After this, patients were usually kept in a weight-bearing cast for approximately 4 weeks. The literature suggests that at 1 year the outcome after early and delayed exercises is similar, with higher wound complications in the early exercise group [24].

The main limitation of this study is its retrospective design. However, combining the results of this study with the literature review might lead to the conclusion that the non- or minimally displaced stable AO-B1.1 or SER-2 type fractures should be treated conservatively and the unstable 
AO-B1.3-B3.3 and SER-4 should be treated operatively [13]. The difficulty lies in determining the best treatment option for the intermediate displaced distal fibular fractures (AO-B1.2). A randomized controlled trial would be necessary to determine the cut-off in displacement to be acceptably treated non-operatively.

The determinants of outcome used in this study were previously reported in the other studies for comparison purposes. The outcome scores used are frequently used scores for ankle fractures and other foot and ankle conditions. Combining three scores gave greater insight into the functional outcome of a large population of patients with an isolated Weber-B ankle fracture. Two observers independently classified the fractures and discussed the individual cases until consensus was met, to achieve the highest possible quality of data.

In conclusion, the current data show that when treatment regimen for Weber-B fractures is based upon dislocation of the distal fibular fragment and fracture type (AO-Weber and Lauge-Hansen), the overall outcome per treatment regime is good to excellent. Shortening the duration of cast immobilization could lead to improved outcome.

Open Access This article is distributed under the terms of the Creative Commons Attribution Noncommercial License which permits any noncommercial use, distribution, and reproduction in any medium, provided the original author(s) and source are credited.

\section{References}

1. Anand N, Klenerman L (1993) Ankle fractures in the elderly: MUA versus ORIF. Injury 24(2):116-120

2. Bauer M, Bergstrom B, Hemborg A, Sandegard J (1985) Malleolar fractures: nonoperative versus operative treatment. A controlled study. Clin Orthop Relat Res 199:17-27

3. Beauchamp CG, Clay NR, Thexton PW (1983) Displaced ankle fractures in patients over 50 years of age. J Bone Joint Surg Br 65(3):329-332

4. Boddenberg U (2004) Healing time of foot and ankle fractures in patients with diabetes mellitus: literature review and report on own cases. Zentralbl Chir 129(6):453-459

5. Broos PL, Bisschop AP (1991) Operative treatment of ankle fractures in adults: correlation between types of fracture and final results. Injury 22(5):403-406

6. Burwell HN, Charnley AD (1965) The treatment of displaced fractures at the ankle by rigid internal fixation and early joint movement. J Bone Joint Surg Br 47(4):634-660

7. Court-Brown CM, Caesar B (2006) Epidemiology of adult fractures: a review. Injury 37(8):691-697

8. Court-Brown CM, McBirnie J, Wilson G (1998) Adult ankle fractures-an increasing problem? Acta Orthop Scand 69(1):43-47

9. Dietrich A, Lill H, Engel T, Schonfelder M, Josten C (2002) Conservative functional treatment of ankle fractures. Arch Orthop Trauma Surg 122(3):165-168

10. Ebraheim NA, Mekhail AO, Gargasz SS (1997) Ankle fractures involving the fibula proximal to the distal tibiofibular syndesmosis. Foot Ankle Int 18(8):513-521
11. Egol KA, Tejwani NC, Walsh MG, Capla EL, Koval KJ (2006) Predictors of short-term functional outcome following ankle fracture surgery. J Bone Joint Surg Am 88(5):974-979

12. Eventov I, Salama R, Goodwin DR, Weissman SL (1978) An evaluation of surgical and conservative treatment of fractures of the ankle in 200 patients. J Trauma 18(4):271-274

13. Gougoulias N, Khanna A, Sakellariou A, Maffulli N (2010) Supination-external rotation ankle fractures: stability a key issue. Clin Orthop Relat Res 468(1):243-251

14. Hahn DM, Colton CL (2001) Malleolar fractures. In: Rüedi TP, Murphy WM (eds) AO Principles of fracture management. Thieme, New York, pp 583-584

15. Kitaoka HB, Alexander IJ, Adelaar RS, Nunley JA, Myerson MS, Sanders M (1994) Clinical rating systems for the ankle-hindfoot, midfoot, hallux, and lesser toes. Foot Ankle Int 15(7):349-353

16. Lauge-Hansen N (1954) Fractures of the ankle. III. Genetic roentgenologic diagnosis of fractures of the ankle. Am J Roentgenol Radium Ther Nucl Med 71(3):456-471

17. Makwana NK, Bhowal B, Harper WM, Hui AW (2001) Conservative versus operative treatment for displaced ankle fractures in patients over 55 years of age. A prospective, randomised study. J Bone Joint Surg Br 83(4):525-529

18. Malka JS, Taillard W (1969) Results of nonoperative and operative treatment of fractures of the ankle. Clin Orthop Relat Res 67:159-168

19. Michelson JD (1995) Fractures about the ankle. J Bone Joint Surg Am 77(1):142-152

20. Olerud C, Molander H (1984) A scoring scale for symptom evaluation after ankle fracture. Arch Orthop Trauma Surg 103(3):190-194

21. Phillips WA, Schwartz HS, Keller CS, Woodward HR, Rudd WS, Spiegel PG, Laros GS (1985) A prospective, randomized study of the management of severe ankle fractures. J Bone Joint Surg Am 67(1):67-78

22. Rowley DI, Norris SH, Duckworth T (1986) A prospective trial comparing operative and manipulative treatment of ankle fractures. J Bone Joint Surg Br 68(4):610-613

23. Stufkens SA, van den Bekerom MP, Kerkhoffs GM, Hintermann B, van Dijk CN (2011) Long-term outcome after 1822 operatively treated ankle fractures: a systematic review of the literature. Injury 42(2):119-127

24. Thomas G, Whalley H, Modi C (2009) Early mobilization of operatively fixed ankle fractures: a systematic review. Foot Ankle Int 30(7):666-674

25. Tile M (2005) Fractures of the ankle. In: Schatzker J, Tile M (eds) The Rationale of operative fracture care. Springer-Verlag, New York, pp 580-581

26. Tunturi T, Kemppainen K, Patiala H, Suokas M, Tamminen O, Rokkanen P (1983) Importance of anatomical reduction for subjective recovery after ankle fracture. Acta Orthop Scand 54(4):641-647

27. Wilson FC Jr, Skilbred LA (1966) Long-term results in the treatment of displaced bimalleolar fractures. J Bone Joint Surg Am 48(6): 1065-1078

28. Yablon IG, Heller FG, Shouse L (1977) The key role of the lateral malleolus in displaced fractures of the ankle. J Bone Joint Surg Am 59(2):169-173

29. Yde J, Kristensen KD (1980) Ankle fractures. Supination-eversion fractures stage II. Primary and late results of operative and non-operative treatment. Acta Orthop Scand 51(4):695-702

30. Yde J, Kristensen KD (1980) Ankle fractures: supination-eversion fractures of stage IV. Primary and late results of operative and non-operative treatment. Acta Orthop Scand 51(6):981-990 